2014

\title{
LibX: The Small but Mighty Button for E-Resource Discovery and Access
}

\author{
Galadriel Chilton \\ University of Connecticut - Storrs, galadriel.chilton@lib.uconn.edu \\ Joelle Thomas \\ University of Connecticut - Storrs, joelle.thomas@lib.uconn.edu
}

Follow this and additional works at: https://opencommons.uconn.edu/libr_pubs

Part of the Collection Development and Management Commons

\section{Recommended Citation}

Chilton, Galadriel and Thomas, Joelle, "LibX: The Small but Mighty Button for E-Resource Discovery and Access" (2014). Published Works. 51.

https://opencommons.uconn.edu/libr_pubs/51 


\title{
LibX: The Small but Mighty Button for E-Resource Discovery and Access
}

\author{
By GALADRIEL CHILTON and JOELLE THOMAS
}

LibX is an open-source browser extension that pushes access to a library's e-resources and services out to users wherever they are on the Internet (e.g. Amazon, Wikipedia, etc.). In Fall 2012, University of Connecticut (UConn) Libraries unveiled their instance of LibX along with homegrown user guides and instructional materials, as well as targeted marketing and promotion efforts. In this session, the presenters described the successes and challenges of UConn's LibX implementation and promotion, as well as an analysis of LibX.

KEYWORDS e-resource access, user behavior, information seeking, open source, information access, resource discovery

\section{BACKGROUND: MEETING OUR USERS WHERE THEY ARE}

One of the challenges libraries face after spending millions of dollars on subscribing and purchasing access to databases and e-journals is figuring out how to extend access to users in their native online habitat. Our referring statistics show that this native online habitat for a significant number of our users is not-despite all of our agonizing over link placement and toplevel links-the library's website. For example, $41 \%$ of our users access our Open URL menu via open websites (Figure 1), 66\% of our users access SpringerLink e-journals via a direct request or open websites (Figure 2), and 65\% of our users arrive at JSTOR content via a direct request or open websites (Figure 3). 
Figure 1: January 2012 through May 2013: Where were our users when they clicked and generated an Open URL menu? Forty-one percent came from open websites (sum of PubMed and Google).

\section{Open URL Resolver Referring Sites = $41 \%$ from open sites}

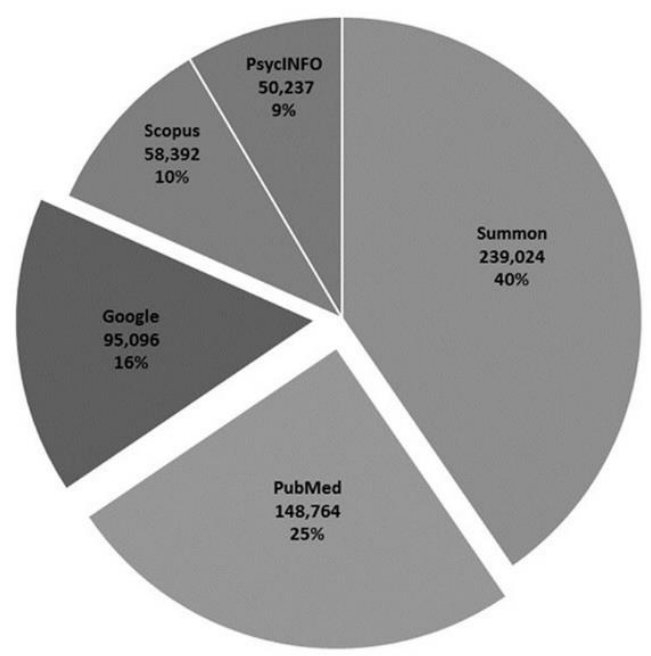

Figure 2: January 2012 through May 2013: Where were our users when they accessed SpringerLink e-journals? Sixty-six percent arrived via a direct request or from open websites (sum of PubMed, Google, and Google Scholar).

\section{Arriving at SpringerLink $=$ $66 \%$ direct or from open sites}

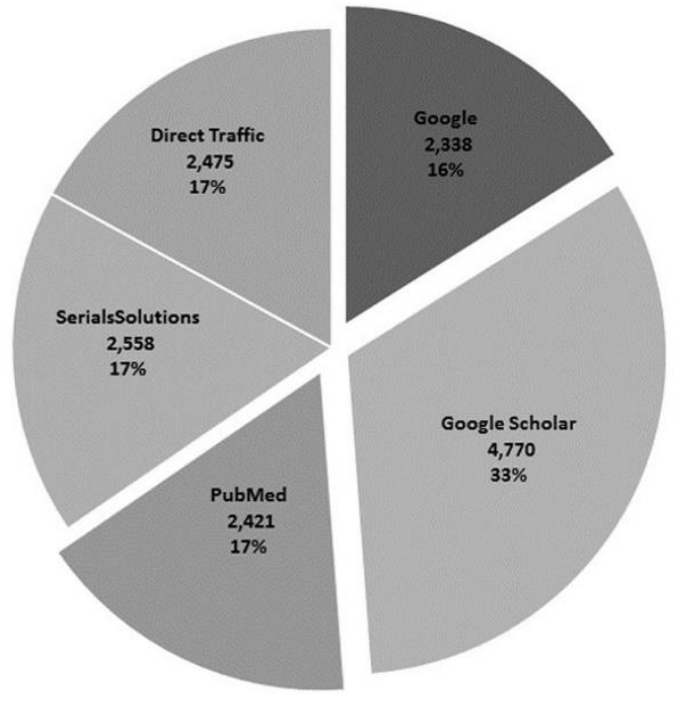


Figure 2: January 2012 through May 2013: How did our users get to JSTOR?

\section{Arriving at JSTOR $=$ $65 \%$ direct or from open websites}

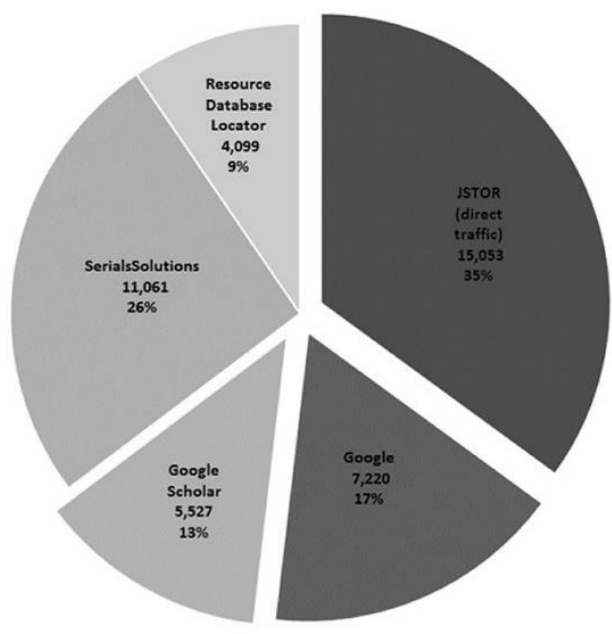

Clearly, if we want users to access library resources, we need to step away from the home page and determine other methods of providing and authenticating access to paid content. Extending access to reflect real user behavior has implications beyond simply ensuring a high return on investment for our expensive e-resources as our ability to meet users' expectations determines an academic libraries' value to them. To be relevant to our users, we must meet their expectations of providing access to the information that they need for their work, with the goal of facilitating successful achievement of their aspirations.

In his February 7, 2013 keynote address at the LYRASIS eGathering, president and founder of The Harwood Institute, Rich Harwood, named four steps required of everyone in the library for libraries to turn outward and be relevant instead of just stating that we are relevant or 
defending relevance:

1. Reflect users' aspirations,

2. Create conditions for community to work,

3. Foster a sense of impact and enduring belief, and

4. Be part of narratives that help us make sense of where we've been, where we are, and where we can go. ${ }^{1}$

With regard to e-resources and access, we might pay particular attention to the first and fourth steps. Rather than thinking of our users from a programmatic or budgetary perspective, can we relate to their personal aspirations and experiences? Do we understand our communities well enough to speak to them in their own language and the things they care about? Can we position ourselves as part of our users' personal narratives? If we are unable to meet our users where they are, when and how they need library resources, we cannot expect to merit more than a footnote in the their personal narratives of information-seeking.

In his April 23 address at the UConn Libraries Spring Forum, Tom Scheinfeldt put it bluntly: "We need to fess up to the fact that our websites may never be destinations in and of themselves for the people we want to serve." ${ }^{2}$ If helping users meet their aspirations includes providing access to our rich collections, and users' information-seeking practices bypass the library's website, then we must ensure that they have direct access to our collections. Our collections should be findable via Google if we want them to be found at all.

\section{A SMALL BUT MIGHTY ASSISTANT}

With these philosophical underpinnings and goals in mind, we determined that one small but effective way to work towards meeting users in their native habitat and helping them achieve their aspirations is via tools, plugins, add-ons, and APIs like LibX. An open-source browser plugin available for Chrome and Firefox, LibX's features include an easy-access search box for library resources, links to library services, dynamic links back to targeted library holdings' 
information for citations and books found on free websites (such as Amazon and Wikipedia), and a "reload current page with EZ Proxy" option for off-campus authentication. With this collection of tools, a user can search library holdings, quickly access services such as interlibrary loan, and take advantage of paid content without ever leaving their native online habitat. There is no need for users to pause in their pursuit of information to discern where on a library's website they should go and what series of many steps are necessary to retrieve needed resources. A user who finds an interesting book in Amazon can discover with a single click whether a copy is available in the library; a user searching Google Scholar can see at a glance what articles are part of the library's subscription packages.

Customizing LibX for our institution proved fairly straightforward. We configured the first iteration of LibX in a couple of hours with search functionality for the e-journal locator, Summon, Google Scholar, UConn's catalog, and WorldCat, as well as links to services and help.

Configuring search functionality for a curated cluster of EBSCO databases took some additional tweaking but was not particularly onerous. Adding links to specific online resources and specifications for our link resolver and proxy was also simple.

The problem with LibX is that users have to know of its existence in order to take advantage of its power. While we added LibX to the image for library computers and let users enjoy much of its might without even being aware of its presence, users certainly do not perform all of their research on library-owned equipment. In order to reach most of our users, we needed to market effectively and convince them of the benefits of installing the software on their personal computers.

\section{MARKETING THE MIGHTY}

Because implementing LibX aligned with an element of the UConn Libraries strategic plan-namely, improving the visibility of online resources - this project met the criteria for submitting a UConn Libraries' Planning Team Proposal that, if approved, included allocation of 
funds for marketing LibX. Our plan was approved and we were allocated $\$ 3,480.00$ to cover the costs for the following marketing efforts:

- Gift certificates for our promotional contest winners,

- An ad in the campus newspaper,

- Table tent ads for the dining halls,

- Two separate two-week ad runs on the campus buses,

Informational fliers, and

- Promotional jump drives containing a link to our in-house LibX installation guide. Not all of these plans came to fruition; ads in the newspaper and on the buses never actually ran.

Marketing also got off to a later start than intended because of technical troubles on LibX's end. Within a few days of our rollout, Chrome changed the way it handles extension installation and caught LibX's creators off-guard. While the issue was eventually resolved, in the meantime we made a series of quick updates to our installation guide to reflect the near-daily changes to what workarounds were required to install LibX in Chrome. Shortly thereafter, Wikipedia temporarily removed its support for $\mathrm{COinS}^{3}$, which LibX relies upon to generate live links to cited content. As with Chrome's installer woes, we could only wait for the problem to be fixed.

We also discovered that before launching the promotional contest, the rules would have to be reviewed and approved by UConn's Office of Compliance and Ethics as well as by UConn's Office of the General Counsel. These are excellent ethical safeguards were also unexpectedly time-consuming. All told, the cascading effects of various delays led to cancelled marketing efforts and greatly delayed creation of in-house promotional videos.

Despite delays and missed opportunities, UConn Libraries' instance of LibX was still live by Fall 2012, and we were able to introduce it to the rest of the library staff through in-person demos and library-wide virtual meetings. This "training the trainers" model allowed for effective 
dissemination of the gospel of LibX. Librarians at UConn Regional campuses introduced their users to LibX as part of the library introduction and instruction sessions. One librarian at the main campus integrated a quick LibX demo into all 29 of her fall and spring nursing instruction sessions.

\section{MEASURING SUCCESS}

Unfortunately, the current iteration of LibX does not provide usage statistics. We have no way of knowing how many UConn users have installed LibX and of those who installed it, how many are actively using it. However, we were able to capture some data more indirectly.

Using stats from the official UConn URL shortener service, we know that there were 115 clicks to a screen capture demo of LibX from our regional campuses, and, from all of UConn, 129 clicks on one particular link to our LibX install site. In a Fall 2012 research workshop attended by 120 grad and post doc students, we handed out flash drives with a short cut to the LibX libguide and gave a short demo of LibX's functionality, after which we collected overwhelmingly positive feedback from attendees; $40 \%$ of them identified LibX as their favorite workshop content. The installation guide that we created and linked from the promotional jump drives has received just under 1,000 hits since its launch.

In addition, our promotional contest collected testimonials from LibX users. Entries included such lines as "LibX has made it easier to access various sources on the UConn Library site and also makes it SO much easier to access articles when I am not on campus!" and "It has made it easier because instead clicking through multiple pages I can simply click on the toolbar and ask for help no problem." All submitted testimonials were highly positive and demonstrated legitimate use of the plugin.

Furthermore, our Open URL referring stats show that between August 2012 and July 2013, users have reached our Open URL resolver menu from Wikipedia 96 times and have reached the menu via LibX functionality 878 times (e.g. clicks on ISBNs, ISSNs, DOI's and PubMed ids that are LibX live links). Numbers to note, but certainly quite small when 
considering that during the same time period, users have reached the Open URL menu nearly 150,000 times via Summon, just under 100,000 via Pubmed, and just over 60,000 times via Google.

\section{CONCLUSION}

LibX is not a perfect solution to our goals of knowing our users' aspirations and providing them with access to needed content via their native online habitats. Despite our efforts to date, it has been adopted by only a very small fraction of UConn users, though many more have likely unwittingly taken advantage of it through its installation on library workstations. However, LibX is just one small tool for achieving these lofty goals, and is still fairly new to UConn. There may never be a single magic bullet for user access to paid content; the best we can do is implement a smorgasbord of effective solutions when we can. Even the smallest of them can be quite mighty.

\section{NOTES}

1. Harwood, R. (2013, February). LYRASIS eGathering Keynote Address. Symposium conducted at the LYRASIS eGathering, online.

2. Scheinfeldt, T. (2013, April). Perspectives and Current Trends in Digital Scholarship. Keynote Address. UConn Libraries Spring Forum.

3. Wikipedia contributors, "Removing COINS Metadata," Wikipedia, The Free Encyclopedia, http://en.wikipedia.org/wiki/Template_talk:Citation/core\#Removing_COINS_metadata (accessed June 3, 2013).

\section{CONTRIBUTOR NOTES}


Galadriel Chilton is the Electronic Resources Management Librarian at the University of Connecticut (galadriel.chilton@lib.uconn.edu).

Joelle Thomas is the Undergraduate User Experience \& Media Technologies Librarian at the University of Connecticut (joelle.thomas@lib.uconn.edu).

This is an electronic version of an article published in:

Chilton, G. \& Thomas, J. (2014). LibX: The small but mighty button for e-resource discovery and access. The Serials Librarian: From the Printed Page to the Digital Age, 66(1-4), 146-152. http://dx.doi.org/10.1080/0361526X.2014.879019. The Serials Librarian: From the Printed Page to the Digital Age is available online at: http://www.tandfonline.com/toc/wser20/current. 